\title{
Refilexiones y miradas sobre el cuerpo en la clase de educación física y el deporte escolar: "vivencias, sueños, anhelos, sentimientos y emociones".
}

Estamos asistiendo a la emergencia de nuevos paradigmas epistemológicos para tratar de entendernos en un mundo que se nos vuelve pequeño en su globalización, así como dificil de comprender en sus múltiples facetas y relatos, en sus imaginarios de sentido, en la vida misma que transcurre en medio de la tecnologia, la exclusión, la pobreza, la opulencia, la desigualdad, y la academia. (Kon-Moción, 2008)

\section{Resumen}

El presente artículo relata la vivencia de un proceso de investigación desarrollado por el grupo Molúfode durante el año 2010, que tuvo como propósito hallar y analizar los sentidos de cuerpo y movimiento que vivencian niños y jóvenes en las clases de Educación Física y las sesiones de Entrenamiento Deportivo escolar del departamento del Huila. En esa intención se caminó por aulas, patios y escenarios deportivos, escudriñando en las vivencias de los estudiantes del Huila, logrando un acercamiento a la realidad entrañada en la clase de Educación Física y el
Eivar Fernando Vargas Polania Candidato a Magister en Educación Grupo de Investigación Molúfode Universidad Surcolombiana
Deportes Escolar con relación al cuerpo y el movimiento, estableciendo reflexiones entre la realidad hallada y los diversos y coloridos fenómenos políticos, sociales, económicos, religiosos y culturales vividos en el mundo.

Palabras clave: cuerpo, movimiento, sentidos, educación física, entrenamiento deportivo.

Sentido de cuerpo.

¿Cuerpo Humano encarnado o Cuerpo Cyborg?

Cuerpo y mundo forman un sistema, pudiendo el cuerpo actuar sobre el mundo sin la mediación del concepto. No en tanto la racionalidad lo habita, puesto que en la motricidad humana (o el cuerpo en acto), la intencionalidad la conduce. (Jaramillo 2008)

En el incesante trasegar de los cuerpos por el mundo, su historia ha sido intervenida por múltiples y variados fenómenos sociales, politicos 
y económicos, también por paradigmas, tabús, realidades, estereotipos e ideas, que han llevado al cuerpo por un vaivén de percepciones, sentidos y formas de concepción, que van desde lo más sublime, hasta lo más reducido, fragmentado y, en muchas ocasiones, de poco significado.

En este sentido, lo que se cree, percibe y representa el cuerpo ha sido motivo de discusión, encuentro, desencuentro, rechazo y armonía, desde los primeros cuerpos que pisaron la tierra hasta nuestras pisadas, es decir, nosotros cuerpos que vivenciamos la actual realidad. Y es esa la intención del presente artículo, hacer parte de un actual encuentro con algunas de las percepciones y sentidos de cuerpo que fluyen en esta veloz modernidad.

Para ello se recorrieron patios, pasillos, aulas y escenarios, escuchando voces y percibiendo las vivencias de niños, niñas y adolescentes, hallando sus sentidos de cuerpo, aclarando que se hace referencia a ellos como cuerpos" y no a "ellos como poseedores de un cuerpo, esta perspectiva de de un yo como cuerpo, nace de la reflexión provocada por el maestro Zubiri, citado por (Sergio, en-acción 4 Algunas miradas sobre el cuerpo, 2007) y quien lanza esta pregunta al mundo: "¿El ser humano tiene o es un cuerpo?". $Y$ continúa manifestando que si la respuesta es tiene un cuerpo, significa que la realidad corporal humana es anexa, es decir que al tener algo significa tener la posibilidad de desprenderse de ese algo anexo, y añade cuestionando que si seríamos capaces de desprendernos de nuestro cuerpo como un algo anexo, para entonces quedamos con lo que se cree que es la esencia que constituye al ser humano según la historia, es decir, la mente, el alma o el espíritu.

Es importante hacer mención que la historia del cuerpo ha estado radicalmente marcada por un dualismo que se ha perpetuado y que tuvo con Platón algunos de sus cimientos más importantes. Este filosofo griego resuelve que el cuerpo no es más que un instrumento al servicio del alma, el cual debe ser adiestrado, controlado y manipulado, pues sus manifestaciones naturales como los deseos, las emociones, los sentimientos, los placeres y la alegría eran consideradas como perturbadoras de la realización del ser humano y obstáculo para la purificación del alma. Esta idea de cuerpo platoniana basada en principios cristianos, los cuales regian en aquella época, continuó su extensión con Aristóteles, discípulo de Platón, quien insistió y aportó al fortalecimiento de un cuerpo dual, fragmentado, subvalorado y al servicio del alma.

Otro teórico de gran transcendencia en la historia del cuerpo fue Descartes, quien trasciende del hombre basado en los principios idealistas, para proponer un ser humano racional, privilegiando en él la razón exaltándola como la verdadera esencia y constitución del ser humano, dejando al cuerpo nuevamente como un instrumento al servicio de la razón, quien es la encargada de dominarlo y manipularlo según sus deseos, intereses y antojos.

En este sentido, Descartes definió al ser humano como "la suma de dos sustancias diferentes que se relacionaban pero que no se combinaban, por un lado la esencia corporal y otra la esencia inteligible o razón" (Sergio, 2007). Es notable como en la manifestación de Descartes permanece el dualismo y la fragmentación de lo que para él significaba el ser humano, a quien nosotros consideramos como un cuerpo. La mencionada idea de ser humano y con ella la de cuerpo, parece que se han perpetuado desde la voz de Descartes hasta nuestros días, algo que lamentablemente ha acarreado la concepción de un ser humano desentrañado y fraccionado, con 
la concepción de cuerpo como objeto, es decir ese algo poseído como instrumento y desposeido de cualquier esencia o dimensión, ese algo vacio, mecánico y al servicio ya sea de la razón o del alma.

Ahora nos acercaremos a algunos sentidos de cuerpo que se respiran en la actualidad, haciendo referencia a manifestaciones emitidas por seres que habitan hoy el mundo y lo comparten con nosotros.

Iniciaremos con unas voces que pondrán en evidencia como algunas de las ideas expresadas por Platón, Aristóteles y Descartes, con relación al cuerpo y que lo situaron como un elemento deshabitado por esencias y dimensiones, instrumentalizado, mecánico, subyugado y subvalorado, aún permanecen y recorren las instituciones educativas del departamento del Huila y hacen parte de la vivencia de la clase de Educación Física y las sesiones de entrenamiento deportivo.

Las voces a las cuales se hace referencia son las de los profesores de Educación Física del departamento del Huila, quienes, durante la entrevista, expresaron que el cuerpo es ${ }^{*} U_{n}$ instrumento mediante el cual surgen todas las expresiones del ser humano; por consiguiente, es fuente de vida, de pensamiento y de movimiento. Por tanto, que hay que cuidarlo y respetarlo*. En el mismo sentido, manifiesta un docente en especial: *que la clase de Educación Física debe propender por un cuerpo que permita la ejecución de movimientos propios de una buena competencia motriz, pues el cuerpo humano está diseñado para ejercitarse, para el esfuerzo físico; pero que debido a los malos hábitos como el sedentarismo y la mala alimentación, el cuerpo ha comenzado a deteriorarse, a enfermarse a devaluarse. Estamos ante la paradoja de pretender un cuerpo que llene nuestras expectativas, pero queremos conseguirlo sentados*.

Es interesante encontrar que para los profesores el cuerpo es un instrumento, palabra que deja avecinar cual es su perspectiva, pues para la Real Academia Española, la palabra instrumento significa; "un conjunto de diversas piezas combinadas adecuadamente para que sirva con determinado objeto en el ejercicio de las artes y oficios".

Desde esta perspectiva, se podría decir que para los docentes el cuerpo es un instrumento o herramienta para el servicio de algo, pero ipara qué o a quién servirá?. Para la purificación del alma, el espíritu o la razón. Ahora por qué hablar de cuidar y respetar el cuerpo como si cuidáramos un objeto, como cuando cuidamos y respetamos el automóvil que poseemos, la motocicleta o los zapatos que usamos y nos sirven para algún objetivo. Será más pertinente y cercano a nuestra humanidad y realidad hablar de cuidarnos y respetarnos como cuerpos en nuestra relación con otros cuerpos en este mundo.

La perspectiva de cuerpo de los profesores no está muy lejana de la poseída por los entrenadores, quienes con relación a su sentido manifestaron que el cuerpo debe responder eficazmente a las acciones motrices, teniendo como apoyo la formación biológica, mental y axiológica, pero que el cuerpo del deportista tiene que marcar la diferencia frente a los otros cuerpos, porque se busca siempre la forma y rendimiento ideal en la disciplina deportiva.

Para profundizar sobre los conceptos emitidos por los entrenadores, éstos dicen que el ser humano cuando está inmerso en un proceso de entrenamiento deportivo; desde el punto de vista 
físico se fortalece con el ejercicio, su corazón es más fuerte, sano y menos graso, su sistema músculo esquelético se vuelve más resistente a los esfuerzos físicos, se retrasa el cansancio o la fatiga, los músculos mueven cada vez cargas mayores; además, la figura humana es más atractiva y armoniosa. Por otro lado la inteligencia y el nivel de concentración aumentan, es más listo, la capacidad lectora se incrementa y mejora su razonamiento lógico matemático. En lo sociohumanístico, la autoestima se eleva, debido a que la persona vive a gusto con su cuerpo, con las posibilidades que le ofrece el deporte para viajar y para hacer amigos.

Un elemento notable en las apreciaciones dadas por los profesores y entrenadores, es el ser humano fragmentado y el cuerpo reducido y limitado a tan solo una dimensión física y biológica, expresada en la realización y ejecución de movimientos visibles y palpables, orientados hacia el fortalecimiento de los músculos, huesos y otros elementos que físicamente lo componen, todo orientado hacia una reducida evolución biológica del cuerpo.

Otro momento interesante en el que se escuchó a los estudiantes y deportistas, fue durante el desarrollo de los talleres, espacios en los que se vivenció, buscando hallar sus más entrañados sentidos sobre el cuerpo. En esta intención, los estudiantes expresaron que su cuerpo es la creación más valiosa que poseen, afirmando que han sido tratados con respeto y con los cuidados necesarios para mantenerse delgados, fuertes, estéticos y sanos, lo cual se ha logrado a través de acciones y objetivos específicos a desarrollar, teniendo como guía un currículo que les permite desarrollar sus capacidades físicas y su musculatura. También manifiestan que gracias al cuerpo se pueden mover, ver y sentir, y que por ello es necesario prepararlo por medio de prácticas deportivas.

Desde su perspectiva y experiencia, los deportistas mencionan que el cuerpo en el entrenamiento es activo, dinámico y trabajado para verse estéticamente bien y obtener un estado fisico óptimo, pues se busca un buen desarrollo de los huesos y la maduración de habilidades, que permitan tener un cuerpo delgado fuerte y saludable, es decir, un cuerpo atlético con buenas capacidades físicas.

Analizando las expresiones de los estudiantes y deportistas, es perceptible como manifiestan un gran aprecio y deseo por ser cuerpos delgados, fuertes y estereotipados bajo esta modernidad que sublima una belleza instaurada en el modelo de cuerpo mercancía, (Morris, 2000) "en donde los cuerpos son rigurosamente inventados como objetos de visión, de forma que el paraíso en la tierra se centra simplemente en el aspecto brillante, voluptuoso y musculado, venerado en Hollywood, como símbolo de perfección". Ahora parece pertinente preguntar por qué niños, niñas y jóvenes del departamento del Huila desean aquella perfección mencionada por Morris, en la que también se encuentran los adultos. ¿Será que la despiadada ideologia capitalista neoliberal tiene algo que ver en esta anhelada perfección? Lamentablemente la respuesta es si, pues han hecho de los cuerpos una mercancia capaz de crear amplias riquezas, que alimentan el fenómeno globalizador capitalista, que ha generado en la sociedad la necesidad y el desenfrenado deseo por lograr el cuerpo sagrado como lo llama (Teves, 2004) "es decir un cuerpo tratado que se remite al sentido de cuidar de si, comer mejor, hacer ejercicios, consumir energéticos, frecuentar gimnasios, en fin, remite a la idea de cómo integrarse a nuevas tribus, cuya incorporación pasa por indumentarias, hábitos 

y actitudes semejantes entres sus miembros, donde cada vez más el mercado se presenta como mediador; ofrece para todos los gustos y poder adquisitivo todo aquello que se precisa, para ser identificado como alguien liberado, para así cuidarse; tenis, cremas protectores, gorras, etc."

Las palabras de Teves muestran como nos enfrentamos a una nueva idea de cuerpo, integrada ya no solo por el racionalismo, sino también alimentada por el consumismo venerado por las fuerzas capitalistas, en donde el alma ha sido excluida, y el cuerpo es ahora exaltado, pero no como integración de todas las esencias y dimensiones humanas, sino como simbolo de mercancia, en la que se centran la ideología capitalista como elemento de sustentación de su poder. En este sentido, (Rey \& Trigo, 2000), manifiestan "que la historia reciente es caracterizada por una corporeidad instrumentalizada en la cual el cuerpo es considerado, en primer momento, como una herramienta más de produccion laboral, un cuerpo que sufre la tension cuidado-explotacion de la produccion".

Todos los planteamientos de los autores, citados anteriormente, presentan una conexión lineal con las clases de Educación Física y las sesiones de entrenamiento deportivo, pues desde las perspectivas de los profesores, entrenadores, estudiantes y deportistas, se encuentra como estas clases y sesiones representan una forma de satisfacer la nueva idea de cuerpo, es decir, aquel cuerpo esbelto, voluptuoso, musculado y atractivo a la subjetividad de otros seres humanos, y que entre en el club de los cuerpos ideales y sanos, en donde estas parecen ser las características de un cuerpo sano y alejado de una temible enfermedad, pues (Sergio, 2007) "hoy parece que todo lo opuesto a la belleza hollywoodense y el erotismo, es sinónimo y sintoma de enfermedad"
Pero no todo es tristeza, desilusión y lamento para el cuerpo, pues existen aun esperanzas de ser reconocidos como somos, como cuerpos complejos inmersos en una interacción infinita y también compleja con el mundo. La esperanza radica en la existencia de teorías y teóricos que rompen con la crueldad del racionalismo, el cristianismo y el capitalismo, y luchan por el encuentro y el reconocimiento, de la verdadera realidad y naturaleza humana, que trasciende la fotográfica mirada de los ojos y escucha lo percibido por otros sentidos, es decir, sentir y percibir aquello que no se ve, aquello intangible que va mucho más allá de lo físico y lo material. Se hace referencia ya no al cuerpo sino a la corporeidad, (Merleau-Ponty, 1956) "que es la experiencia de ser cuerpo en el mundo, en donde ya no se habla de de una parte de la persona sino de toda ella, "esa persona que vive, siente, piensa, hace cosas, se desplaza, que crece, se emociona, se relaciona con otras personas y con el mundo que le rodea" (Zubiri, 1988) y "que a partir de esas relaciones construye su propio mundo significativo y da sentido a su vida".

Es necesario empezar a buscar y crear formas de concebir el cuerpo, como también el mundo y la formar de vivir en él. Formas que ojalá busquen la complejidad y armonía dialógica, formas en las que no solo seamos reconocidos como cuerpo y alma, cuerpo y razón, carne, huesos y espíritu, sino como cuerpos entrañados de sentimientos, emociones, alegrias, deseos, anhelos, sueños, cultura, política, sociedad, intelecto, amor, tristezas, frustraciones, dolor, temores, opresión, sufrimiento, en fin, todo lo que expresamos como corporeidad humana en el mundo. Esto es algo que no, parece dificil, por eso el consejo de (Rodriguez, 2001) es muy pertinente, invitándonos "que lo que tenemos que hacer es mudar nuestra manera de comprendernos y comprender el mundo" y con ello nuestra forma de vernos y sentir el mundo, otros caminos para 
la Educación Física, pues según (Sergio, 2007) la Educación Física despunta del dualismo antropológico cartesiano y camina hacia la creación del hombre-máquina"

\section{Sentido de movimiento.}

¿Encuentro entre la complejidad y trascendencia real-anhelada, y el imperante, simple y reducido dualismo $i$

Un sujeto que se mueve, es un ser que decide hacer contacto con lo que le rodea a través de la motricidad humana, es un ser que empieza el camino hacia su humanización, libertad y emancipación, exponiendo y revelando su subjetividad ante el contexto y el universo; contacto que será posible a través de un movimiento centrífugo. Es decir, un movimiento multidimensional, que nace en lo más interno del ser y que estará encarnado en la motricidad humana; por consiguiente, es un movimiento que trascenderá de lo visiblemente mecánico y físico hacia lo intangible, expresando todo lo que siente, piensa, desea, anhela, carece, quiere y hace; vivenciando millones de experiencias e interactuando con millones de seres y variadas formas de vida, en un mundo colorido y dinámico"

(Molúfode 2009)

El movimiento humano, tema que hoy interesa, hace parte de la manifestación y vivenciación de la corporeidad por parte de la humanidad Por eso, ha sido un acompañante durante toda su existencia, situación que lo convirtió en un elemento altamente sensible, frente a todas las concepciones con respecto al ser humano, el cuerpo, el área de Educación Física y el entrenamiento deportivo, entre otros. Esta situación hizo que el movimiento fuera una víctima más de todas las laceraciones, la fragmentación y la subordinación, por las cuales ha pasado el ser humano y el cuerpo, viéndose en muchos momentos de la historia, reducido, excluido y despojado de todo su colorido y complejidad en la realidad humana.

En este sentido es importante mencionar que durante la época del idealismo y del cristianismo, los movimientos relacionados con la alegria, el placer, los deseos y los anhelos, fueron considerados como pecaminosos y motivos de condenación social y divina.

Posteriormente en tiempos del auge del racionalismo científico, se hizo sublime a la razón y el intelecto, lo cual trajo serias consecuencias para el movimiento humano, pues según (Sergio \& Toro, 2005) "esta perspectiva de mundo, conocimiento y cuerpo desde el paradigma cartesiano, significó darle a todo lo que significaba un trabajo físico, el carácter de trabajo de segunda clase". Algo que significó la subordinacion total del movimiento humano frente a lo que, según en la epoca, se creía la razón. Esto no fue mas que una falacia, pues es imposible razonar sin mover la complejidad humana y todo lo que ella entraña.

Pero caminamos en los presentes años, en los cuales ha nacido el deseo de realizar un acercamiento al significado de movimiento en la realidad de la clase de Educación Física y el entrenamiento deportivo en el departamento del Huila, para ello fue necesario hacer inmersión en estas prácticas e interactuar con los protagonistas de ellas para dar cumplimiento a deseo mencionado. Pero antes de escuchar las voces de los protagonistas, es importante resaltar que anteriormente se mostró como el dualismo cartesiano, la subvaloración y el reduccionismo, así como la tendencia deportivista que hacen 
parte de la realidad actual de la Educación Física y el entrenamiento deportivo escolar, inciden ampliamente en la realidad del cuerpo, lo cual desde ya presupone una incidencia también en el la realidad del movimiento humano en la escuela.

En el deseo de hallar la realidad y el sentido del movimiento en la clase de Educación Física, se interactuó con los docentes encontrando como sienten el movimiento como un elemento fundamental para el proceso y el desarrollo integral del educando tanto físico, psicológico, motriz, social, etc., a tal punto que se le considera la base y el principio de los diferentes hábitos hacia un estilo de vida plena, convirtiéndose este en el eje imprescindible de la clase de Educación Fisica. También expresaron que los movimientos en la clase son expresiones corporales, del entorno y hacia el entorno, con la intervención, ayuda y orientación de educadores físicos, quienes con su conocimiento favorecen la espontaneidad y la maduración motriz de los patrones básicos del movimiento.

Por su parte, los entrenadores dejaron claro que el movimiento es un factor determinante para la adquisición de los gestos técnicos y de la forma deportiva en general, con las cuales se construye cada una de las sesiones del entrenamiento como instrumento para conseguir los objetivos propuestos.

Desde una perspectiva cercana a la de los docentes y entrenadores, los estudiantes le dan gran importancia al movimiento, argumentando que a través de la Educación Física se adquiere disciplina teniendo en cuenta que para cada actividad fisica se requiere del movimiento que se logra con trabajo, disciplina y con un grado de exigencia personal. Para otros, se convierte en el elemento que les permite desempeñar acciones de la vida cotidiana y recomiendan variedad de tareas para evitar la monotonía.

Desde sus vivencias los deportistas expresaron que el movimiento en el entrenamiento significa preparación y formación dentro de una disciplina deportiva, diferenciando el ser humano y la máquina. El movimiento para ellos es el principal gestor de la fuerza y la evolución muscular y lo consideran también como la base de la técnica, acompañado de factores que implican un desarrollo deportivo.

Reflexionando sobre los sentidos manifestados por los profesores, los entrenadores, los estudiantes y los deportistas, nacen las siguientes preguntas: ¿Cuando nos movemos, sólo lo hacemos con nuestros huesos, músculos e impulsados por nuestro sistema nervioso? ¿Y cuando pateamos o lanzamos un balón no lo hacemos también con alegría, emoción, entusiasmo, deseo, o con tristeza, frustración, rabia, desespero o dolor? ¿Sera que estos movimientos, además de los impulsos emitidos por las neuronas eferentes, no van motivados por nuestras intenciones y conciencia?

Estas preguntas podrian encontrar sus respuestas en la teoria de la motricidad humana, la cual es definida como "el cuerpo en acto y la energía expresada para el movimiento centrifugo y centrípeto de la personalización" (Sergio \& Toro, 2005). En otras palabras, es la energía intencional para el movimiento de trascendencia, que se convierte en acción, es decir, que la motricidad es la energía expresada para la acción de superación.

Al parecer la Educación Física y el Entrenamiento Deportivo, se han desarrollado sobre una concepción de movimiento bastante reducida y radicada en lo visible y palpable, 
obedeciendo a la mirada que les propina la competencia, el estándar, el rendimiento y el ansia por la marca y la técnica perfecta, debido a "que todo y todos estamos tildados de siglos de racionalismo, de objetividad del conocimiento en departamentos estancos que nos están impidiendo el acercamiento a la diversidad de modos de conocer que posee el ser humano en su complejidad" (Sergio).

En este sentido y referente al movimiento, el grupo de investigación (Molúfode, 2009) expresa "que un sujeto que se mueve, es un ser que decide hacer contacto con lo que le rodea a través de la motricidad humana, entonces será un ser que empieza el camino hacia su humanización, libertad y emancipación, exponiendo y revelando su subjetividad ante el contexto y el universo; contacto que será posible a través de un movimiento centrifugo. Es decir, un movimiento multidimensional, que nace en lo más interno del ser y que estará encarnado en la motricidad humana; por consiguiente, es un movimiento que trascenderá de lo visiblemente mecánico y físico hacia lo intangible, expresando todo lo que siente, piensa, desea, anhela, carece, quiere y hace; vivenciando millones de experiencias e interactuando con millones de seres y variadas formas de vida, en un mundo colorido y dinámico".

En los párrafos anteriores se ha mostrado, como existen otras posibilidades de movimiento, posibilidades que resisten al racionalismo, al capitalismo y a la ceguera ante la complejidad humana. Se refirieren a formas que reclaman por lo que verdaderamente encarna el movimiento humano, por aquello que sentimos y que llevamos cuando nos movemos, elementos que van mucho más allá de un simple movimiento en el espacio, pues estamos encarnados de conciencia, intención y motivación, poniendo en acción en el universo, todo el arcoíris de sentimientos y emociones que nos integran como seres complejos.

Si no fuera así, se podría pensar que el lector que lee estas líneas, solo utiliza sus estructuras internas necesarias para realizar dicha lectura, pero es claro que si estás leyendo esto, es porque algo tc motiva, algo te impulsa a leer, y tal vez te interesa leerlo, y desde tu conciencia y reflexión puedes estar de acuerdo con lo que aqui se expone, o por el contrario puedes tener resistencia, puedes generar afecto y simpatía, así como rechazo o desagrado. Entonces, ¿dónde está la conciencia, la reflexión, la motivación, los deseos, los sueños, los anhelos y las tristezas en el movimiento vivenciado en la clase de Educación Física y el Entrenamiento Deportivo? $\mathrm{O}$ ¿Por qué negamos su existencia cuando lo sentimos? o ¿Por qué no lo queremos reconocer?. Parece que ha llegado el momento de empezar a invitar a entrenadores, profesores, estudiantes y deportistas, a que inicien a reconocerse y sentirse como seres humanos y reconocer su complejidad, corporeidad y motricidad la cual los encarna y han negado durante años.

Luego de reflexionar un poco sobre el sentido de movimiento, el deseo es descubrir qué tipos de movimientos son los que más se desarrollan en la clase y el entrenamiento. En este sentido, un profesor en especial manifestó que: * En la clase de Educación Física se desarrollan movimientos naturales de los estudiantes, llegando a un acuerdo desde una construcción colectiva con clara influencia cultural, ya sean orientados a manifestaciones deportivas o expresiones motrices propias, se debe estimular la independencia segmentaria y, en general, afianzar muchas conductas del esquema corporal y de las conductas motrices de base. Sin embargo, considero que hay una subutilización del 
potencial motriz que reclama atención como la percepción rítmica* ${ }^{*}$

Desde otra perspectiva, los entrenadores consideran que los movimientos en cada sesión se realizan de una manera consciente y estructurada, son movimientos finos con un alto grado de técnica y acompañado de capacidades fisicas, a través de una actividad integral que permite una acción, una experiencia motriz especifica que, al ser llevada a través de procesos, permite llegar al perfeccionamiento y maduración del ser humano.

Desde sus experiencias los estudiantes expresaron que, en muchos casos, existe monotonia en las actividades y no hay mucho tiempo para el goce de la clase, para otros la clase se desarrolla dentro de lo normal, calentamiento, movimientos básicos y una actividad central, todo es dirigido estrictamente por el profesor sin que exista consenso con el estudiante. Mientras que para los deportistas los movimientos son específicos del deporte, pues son elementos que les ayudan para llegar al máximo rendimiento y mediante un trabajo planificado se alcanzar una técnica específica. Con este trabajo se pretende llegar al máximo grado de perfeccionamiento y alcanzar objetivos trazados.

Nuevamente se encuentra como en las clases de Educación Física y las sesiones de entrenamiento deportivo se persigue desesperadamente (Sergio, 2007) "el cuerpo-cyborg que es la función máquina organismo y el ideal en esta postmodernidad", ya que han quedado evidencias de cómo están impregnadas de la tendencia deportiva e integrada por actividades que fomentan el desarrollo de movimientos relacionados con las capacidades físicas, lo cual hace parte de la gama de la vivencia de la corporeidad. Pero es tan solo una parte de la compleja motricidad humana. En este sentido, (Molúfode, 2009), en términos generales, expresa "Que en las expresiones motrices, se integran una multiplicidad de movimientos que trascienden a acciones politicas, sociales y culturales en las cuales interactúa el sujeto con el otro y el contexto". Con simpatía por las palabras de Molúfode, es importante aportar que las expresiones motrices son la forma en la que el ser humano vivencia y expande su corporeidad en el universo, encarnado en la conciencia, la reflexión, la emancipación, el amor por la vida y la libertad.

Las palabras de la profesora Rubiela y del Grupo de Investigación Molúfode invitan a la reflexión sobre la reducida dinámica en la que vive la Educación Física y el Deporte Escolar, pues se ha evidenciado como el cuerpo y el movimiento humano no sólo se remite a ese ejercicio físico y visible, concentrado en las ansias de aumentar la resistencia, la fuerza, la disciplina, la reciedumbre, la velocidad, entre otras. ¿Por qué no se busca también el amor, la convivencia, el liderazgo?, ¿por qué no se mencionan las emociones y los sentimientos como integrantes de la complejidad humana? ¿Por qué se desprecian las tristezas, las alegrias, los sueños y las frustraciones en las clases y las sesiones de entrenamiento? En fin, ¿Por qué la ceguera ante la compleja motricidad humana?

Las voces a las cuales se hace referencia son las de los profesores de Educación Física del departamento del Huila, quienes, durante la entrevista, expresaron que el cuerpo es ${ }^{*} U_{n}$ instrumento mediante el cual surgen todas las expresiones del ser humano; por consiguiente, es fuente de vida, de pensamiento y de movimiento. Por tanto, que hay que cuidarlo y respetarlo*. En el mismo sentido, manifiesta un docente en especial: *que la clase de Educación Física debe propender por un cuerpo que permita 
la ejecución de movimientos propios de una buena competencia motriz, pues el cuerpo humano está diseñado para ejercitarse, para $\mathrm{cl}$ esfuerzo físico; pero que debido a los malos hábitos como el sedentarismo y la mala alimentación, el cuerpo ha comenzado a deteriorarse, a enfermarse a devaluarse. Estamos ante la paradoja de pretender un cuerpo que llene nuestras expectativas, pero queremos conseguirlo sentados*.

Es interesante encontrar que para los profesores el cuerpo es un instrumento, palabra que deja avecinar cual es su perspectiva, pues para la Real Academia Española, la palabra instrumento significa; "un conjunto de diversas piezas combinadas adecuadamente para que sirva con determinado objeto en el ejercicio de las artes y oficios".

Desde esta perspectiva, se podría decir que para los docentes el cuerpo es un instrumento o herramienta para el servicio de algo, pero ipara qué o a quién servirá?. Para la purificación del alma, el espíritu o la razón. Ahora por qué hablar de cuidar y respetar el cuerpo como si cuidáramos un objeto, como cuando cuidamos y respetamos el automóvil que poseem-os, la motocicleta o los zapatos que usamos y nos sirven para algún objetivo. Será más pertinente y cercano a nuestra humanidad y realidad hablar de cuidarnos y respetarnos como cuerpos en nuestra relación con otros cuerpos en este mundo.

La perspectiva de cuerpo de los profesores no está muy lejana de la poseída por los entrenadores, quienes con relación a su sentido manifestaron que el cuerpo debe responder eficazmente a las acciones motrices, teniendo como apoyo la formación biológica, mental y axiológica, pero que el cuerpo del deportista tiene que marcar la diferencia frente a los otros cuerpos, porque se busca siempre la forma y rendimiento ideal en la disciplina deportiva.

Para profundizar sobre los conceptos emitidos por los entrenadores, éstos dicen que el ser humano cuando está inmerso en un proceso de entrenamiento deportivo; desde el punto de vista físico se fortalece con el ejercicio, su corazón es más fuerte, sano y menos graso, su sistema músculo esquelético se vuelve más resistente a los esfuerzos físicos, se retrasa el cansancio o la fatiga, los músculos mueven cada vez cargas mayores; además, la figura humana es más atractiva y armoniosa. Por otro lado la inteligencia y el nivel de concentración aumentan, es más listo, la capacidad lectora se incrementa y mejora su razonamiento lógico matemático. En lo sociohumanístico, la autoestima se eleva, debido a que la persona vive a gusto con su cuerpo, con las posibilidades que le ofrece el deporte para viajar y para hacer amigos.

Un elemento notable en las apreciaciones dadas por los profesores y entrenadores, es el ser humano fragmentado y el cuerpo reducido y limitado a tan solo una dimensión física y biológica, expresada en la realización y ejecución de movimientos visibles y palpables, orientados hacia el fortalecimiento de los músculos, huesos y otros elementos que fisicamente lo componen, todo orientado hacia una reducida evolución biológica del cuerpo.

Otro momento interesante en el que se escuchó a los estudiantes y deportistas, fue durante el desarrollo de los talleres, espacios en los que se vivenció, buscando hallar sus más entrañados sentidos sobre el cuerpo. En esta intención, los estudiantes expresaron que su cuerpo es la creación más valiosa que poseen, afirmando que han sido tratados con respeto y con los cuidados 
necesarios para mantenerse delgados, fuertes, estéticos y sanos, lo cual se ha logrado a través de acciones y objetivos específicos a desarrollar, teniendo como guia un currículo que les permite desarrollar sus capacidades fisicas y su musculatura. También manifiestan que gracias al cuerpo se pueden mover, ver y sentir, y que por ello es necesario prepararlo por medio de prácticas deportivas.

Desde su perspectiva y experiencia, los deportistas mencionan que el cuerpo en el entrenamiento es activo, dinámico y trabajado para verse estéticamente bien y obtener un estado físico óptimo, pues se busca un buen desarrollo de los huesos y la maduración de habilidades, que permitan tener un cuerpo delgado fuerte y saludable, es decir, un cuerpo atlético con buenas capacidades físicas.

Analizando las expresiones de los estudiantes y deportistas, es perceptible como manifiestan un gran aprecio y deseo por ser cuerpos delgados, fuertes y estereotipados bajo esta modernidad que sublima una belleza instaurada en el modelo de cuerpo mercancia, (Morris, 2000) "en donde los cuerpos son rigurosamente inventados como objetos de visión, de forma que el paraíso en la tierra se centra simplemente en el aspecto brillante, voluptuoso y musculado, venerado en Hollywood, como símbolo de perfección". Ahora parece pertinente preguntar por qué niños, niñas y jóvenes del departamento del Huila desean aquella perfección mencionada por Morris, en la que también se encuentran los adultos. ¿Será que la despiadada ideologia capitalista neoliberal tiene algo que ver en esta anhelada perfección? Lamentablemente la respuesta es sí, pues han hecho de los cuerpos una mercancía capaz de crear amplias riquezas, que alimentan el fenomeno globalizador capitalista, que ha generado en la sociedad la necesidad y el desenfrenado deseo por lograr el cuerpo sagrado como lo llama (Teves, 2004) "es decir un cuerpo tratado que se remite al sentido de cuidar de sí, comer mejor, hacer ejercicios, consumir energéticos, frecuentar gimnasios, en fin, remite a la idea de cómo integrarse a nuevas tribus, cuya incorporación pasa por indumentarias, hábitos $y$ actitudes semejantes entres sus miembros, donde cada vez más el mercado se presenta como mediador; ofrece para todos los gustos y poder adquisitivo todo aquello que se precisa, para ser identificado como alguien liberado, para así cuidarse; tenis, cremas protectores, gorras, etc."

Las palabras de Teves muestran como nos enfrentamos a una nueva idea de cuerpo, integrada ya no solo por el racionalismo, sino también alimentada por el consumismo venerado por las fuerzas capitalistas, en donde el alma ha sido excluida, y el cuerpo es ahora exaltado, pero no como integración de todas las esencias y dimensiones humanas, sino como símbolo de mercancía, en la que se centran la ideología capitalista como elemento de sustentación de su poder. En este sentido, (Rey \& Trigo, 2000), manifiestan "que la historia reciente es caracterizada por una corporeidad instrumentalizada en la cual el cuerpo es considerado, en primer momento, como una herramienta más de produccion laboral, un cuerpo que sufre la tension cuidado-explotacion de la produccion".

Todos los planteamientos de los autores, citados anteriormente, presentan una conexión lineal con las clases de Educación Física y las sesiones de entrenamiento deportivo, pues desde las perspectivas de los profesores, entrenadores, estudiantes y deportistas, se encuentra como estas clases y sesiones representan una forma de satisfacer la nueva idea de cuerpo, es decir, aquel cuerpo esbelto, voluptuoso, musculado y 
atractivo a la subjetividad de otros seres humanos, y que entre en el club de los cuerpos ideales y sanos, en donde estas parecen ser las características de un cuerpo sano y alejado de una temible enfermedad, pues (Sergio, 2007) "hoy parece que todo lo opuesto a la belleza hollywoodense y el erotismo, es sinónimo y síntoma de enfermedad"

Pero no todo es tristeza, desilusión y lamento para el cuerpo, pues existen aun esperanzas de ser reconocidos como somos, como cuerpos complejos inmersos en una interacción infinita y también compleja con el mundo. La esperanza radica en la existencia de teorías y teóricos que rompen con la crueldad del racionalismo, el cristianismo y el capitalismo, y luchan por el encuentro y el reconocimiento, de la verdadera realidad y naturaleza humana, que trasciende la fotográfica mirada de los ojos y escucha lo percibido por otros sentidos, es decir, sentir y percibir aquello que no se ve, aquello intangible que va mucho más allá de lo físico y lo material. Se hace referencia ya no al cuerpo sino a la corporeidad, (Merleau-Ponty, 1956) "que es la experiencia de ser cuerpo en el mundo, en donde ya no se habla de de una parte de la persona sino de toda ella, "esa persona que vive, siente, piensa, hace cosas, se desplaza, que crece, se emociona, se relaciona con otras personas y con el mundo que le rodea" (Zubiri, 1988) y "que a partir de esas relaciones construye su propio mundo significativo y da sentido a su vida".

Es necesario empezar a buscar y crear formas de concebir el cuerpo, como también el mundo y la formar de vivir en él. Formas que ojalá busquen la complejidad y armonía dialógica, formas en las que no solo seamos reconocidos como cuerpo y alma, cuerpo y razón, carne, huesos y espíritu, sino como cuerpos entrañados de sentimientos, emociones, alegrías, deseos, anhelos, sueños, cultura, política, sociedad, intelecto, amor, tristezas, frustraciones, dolor, temores, opresión, sufrimiento, en fin, todo lo que expresamos como corporeidad humana en el mundo. Esto es algo que no parece difícil, por eso el consejo de (Rodriguez, 2001) es muy pertinente, invitándonos "que lo que tenemos que hacer es mudar nuestra manera de comprendernos y comprender el mundo" y con ello nuestra forma de vernos y sentir el mundo, otros caminos para la Educación Física, pues según (Sergio, 2007) la Educación Física despunta del dualismo antropológico cartesiano y camina hacia la creación del hombre-máquina"

Sentido de movimiento.

¿Encuentro entre la complejidad y trascendencia real-anhelada, y el imperante, simple y reducido dualismoi

Un sujeto que se mueve, es un ser que decide hacer contacto con lo que le rodea a través de la motricidad humana, es un ser que empieza el camino hacia su humanización, libertad y emancipación, exponiendo y revelando su subjetividad ante el contexto y el universo; contacto que será posible a través de un movimiento centrifugo. Es decir, un movimiento multidimensional, que nace en lo más interno del ser y que estará encarnado en la motricidad humana; por consiguiente, es un movimiento que trascenderá de lo visiblemente mecánico y físico hacia lo intangible, expresando todo lo que siente, piensa, desea, anhela, carece, quiere y hace; vivenciando millones de experiencias e interactuando con millones de seres y variadas formas de vida, en un mundo colorido y dinámico"

(Molúfode 2009)

El movimiento humano, tema que hoy interesa, hace parte de la manifestación y vivenciación de 
la corporeidad por parte de la humanidad Por eso, ha sido un acompañante durante toda su existencia, situación que lo convirtió en un elemento altamente sensible, frente a todas las concepciones con respecto al ser humano, el cuerpo, el área de Educación Física y el entrenamiento deportivo, entre otros. Esta situación hizo que el movimiento fuera una víctima más de todas las laceraciones, la fragmentación y la subordinación, por las cuales ha pasado el ser humano y el cuerpo, viéndose en muchos momentos de la historia, reducido, excluido y despojado de todo su colorido y complejidad en la realidad humana.

En este sentido es importante mencionar que durante la época del idealismo y del cristianismo, los movimientos relacionados con la alegría, el placer, los deseos y los anhelos, fueron considerados como pecaminosos y motivos de condenación social y divina.

Posteriormente en tiempos del auge del racionalismo cientifico, se hizo sublime a la razón y el intelecto, lo cual trajo serias consecuencias para el movimiento humano, pues según (Sergio \& Toro, 2005) "esta perspectiva de mundo, conocimiento y cuerpo desde el paradigma cartesiano, significó darle a todo lo que significaba un trabajo físico, el carácter de trabajo de segunda clase". Algo que significó la subordinacion total del movimiento humano frente a lo que, según en la epoca, se creía la razón. Esto no fue mas que una falacia, pues es imposible razonar sin mover la complejidad humana y todo lo que ella entraña.

Pero caminamos en los presentes años, en los cuales ha nacido el deseo de realizar un acercamiento al significado de movimiento en la realidad de la clase de Educación Fisica y el entrenamiento deportivo en el departamento del
Huila, para ello fue necesario hacer inmersión en estas prácticas e interactuar con los protagonistas de ellas para dar cumplimiento a deseo mencionado. Pero antes de escuchar las voces de los protagonistas, es importante resaltar que anteriormente se mostró como el dualismo cartesiano, la subvaloración y el reduccionismo, así como la tendencia deportivista que hacen parte de la realidad actual de la Educación Física y el entrenamiento deportivo escolar, inciden ampliamente en la realidad del cuerpo, lo cual desde ya presupone una incidencia también en el la realidad del movimiento humano en la escuela.

En el deseo de hallar la realidad y el sentido del movimiento en la clase de Educación Física, se interactuó con los docentes encontrando como sienten el movimiento como un elemento fundamental para el proceso y el desarrollo integral del educando tanto físico, psicológico, motriz, social, etc., a tal punto que se le considera la base y el principio de los diferentes hábitos hacia un estilo de vida plena, convirtiéndose este en el eje imprescindible de la clase de Educación Física. También expresaron que los movimientos en la clase son expresiones corporales, del entorno y hacia el entorno, con la intervención, ayuda y orientación de educadores físicos, quienes con su conocimiento favorecen la espontaneidad y la maduración motriz de los patrones básicos del movimiento.

Por su parte, los entrenadores dejaron claro que el movimiento es un factor determinante para la adquisición de los gestos técnicos y de la forma deportiva en general, con las cuales se construye cada una de las sesiones del entrenamiento como instrumento para conseguir los objetivos propuestos.

Desde una perspectiva cercana a la de los 
docentes y entrenadores, los estudiantes le dan gran importancia al movimiento, argumentando que a través de la Educación Físiça se adquiere disciplina teniendo en cuenta que para cada actividad física se requiere del movimiento que se logra con trabajo, disciplina y con un grado de exigencia personal. Para otros, se convierte en el elemento que les permite desempeñar acciones de la vida cotidiana y recomiendan variedad de tareas para evitar la monotonía.

Desde sus vivencias los deportistas expresaron que el movimiento en el entrenamiento significa preparación y formación dentro de una disciplina deportiva, diferenciando el ser humano y la máquina. El movimiento para ellos es el principal gestor de la fuerza y la evolución muscular y lo consideran también como la base de la técnica, acompañado de factores que implican un desarrollo deportivo.

Reflexionando sobre los sentidos manifestados por los profesores, los entrenadores, los estudiantes y los deportistas, nacen las siguientes preguntas: ¿Cuando nos movemos, sólo lo hacemos con nuestros huesos, músculos e impulsados por nuestro sistema nervioso? iY cuando pateamos o lanzamos un balón no lo hacemos también con alegria, emoción, entusiasmo, deseo, o con tristeza, frustración, rabia, desespero o dolor? ¿Sera que estos movimientos, además de los impulsos emitidos por las neuronas eferentes, no van motivados por nuestras intenciones y conciencia?

Estas preguntas podrian encontrar sus respuestas en la teoría de la motricidad humana, la cual es definida como "el cuerpo en acto y la energía expresada para el movimiento centrífugo y centripeto de la personalización" (Sergio \& Toro, 2005). En otras palabras, es la energía intencional para el movimiento de trascendencia, que se convierte en acción, es decir, que la motricidad es la energía expresada para la acción de superación.

Al parecer la Educación Física y el Entrenamiento Deportivo, se han desarrollado sobre una concepción de movimiento bastante reducida y radicada en lo visible y palpable, obedeciendo a la mirada que les propina la competencia, el estándar, el rendimiento y el ansia por la marca y la técnica perfecta, debido a "que todo y todos estamos tildados de siglos de racionalismo, de objetividad del conocimiento en departamentos estancos que nos están impidiendo el acercamiento a la diversidad de modos de conocer que posee el ser humano en su complejidad" (Sergio).

En este sentido y referente al movimiento, el grupo de investigación (Molúfode, 2009) expresa "que un sujeto que se mueve, es un ser que decide hacer contacto con lo que le rodea a través de la motricidad humana, entonces será un ser que empieza el camino hacia su humanización, libertad y emancipación, exponiendo y revelando su subjetividad ante el contexto y el universo; contacto que será posible a través de un movimiento centrifugo. Es decir, un movimiento multidimensional, que nace en lo más interno del ser y que estará encarnado en la motricidad humana; por consiguiente, es un movimiento que trascenderá de lo visiblemente mecánico y fisico hacia lo intangible, expresando todo lo que siente, piensa, desea, anhela, carece, quiere y hace; vivenciando millones de experiencias e interactuando con millones de seres y variadas formas de vida, en un mundo colorido y dinámico".

En los párrafos anteriores se ha mostrado, como existen otras posibilidades de movimiento, posibilidades que resisten al racionalismo, al capitalismo y a la ceguera ante la complejidad 
humana. Se refirieren a formas que reclaman por lo que verdaderamente encarna el movimiento humano, por aquello que sentimos y que llevamos cuando nos movemos, elementos que van mucho más allá de un simple movimiento en el espacio, pues estamos encarnados de conciencia, intención y motivación, poniendo en acción en el universo, todo el arcoíris de sentimientos y emociones que nos integran como seres complejos.

Si no fuera así, se podría pensar que el lector que lee estas líneas, solo utiliza sus estructuras internas necesarias para realizar dicha lectura, pero es claro que si estás leyendo esto, es porque algo te motiva, algo te impulsa a leer, y tal vez te interesa leerlo, y desde tu conciencia y reflexión puedes estar de acuerdo con lo que aquí se expone, o por el contrario puedes tener resistencia, puedes generar afecto y simpatia, así como rechazo o desagrado. Entonces, ¿dónde está la conciencia, la reflexión, la motivación, los deseos, los sueños, los anhelos y las tristezas en el movimiento vivenciado en la clase de Educación Física y el Entrenamiento Deportivo? O ¿Por qué negamos su existencia cuando lo sentimos? ¡ ¿Por qué no lo queremos reconocer?. Parece que ha llegado el momento de empezar a invitar a entrenadores, profesores, estudiantes y deportistas, a que inicien a reconocerse y sentirse como seres humanos y reconocer su complejidad, corporeidad y motricidad la cual los encarna y han negado durante años.

Luego de reflexionar un poco sobre el sentido de movimiento, el deseo es descubrir qué tipos de movimientos son los que más se desarrollan en la clase y el entrenamiento. En este sentido, un profesor en especial manifestó que: ${ }^{\star} E n$ la clase de Educación Física se desarrollan movimientos naturales de los estudiantes, llegando a un acuerdo desde una construcción colectiva con clara influencia cultural, ya sean orientados a manifestaciones deportivas o expresiones motrices propias, se debe estimular la independencia segmentaria y, en general, afianzar muchas conductas del esquema corporal y de las conductas motrices de base. Sin embargo, considero que hay una subutilización del potencial motriz que reclama atención como la percepción ritmica*.

Desde otra perspectiva, los entrenadores consideran que los movimientos en cada sesión se realizan de una manera consciente y estructurada, son movimientos finos con un alto grado de técnica y acompañado de capacidades físicas, a través de una actividad integral que permite una acción, una experiencia motriz especifica que, al ser llevada a través de procesos, permite llegar al perfeccionamiento $y$ maduración del ser humano.

Desde sus experiencias los estudiantes expresaron que, en muchos casos, existe monotonía en las actividades y no hay mucho tiempo para el goce de la clase, para otros la clase se desarrolla dentro de lo normal, calentamiento, movimientos básicos y una actividad central, todo es dirigido estrictamente por el profesor sin que exista consenso con el estudiante. Mientras que para los deportistas los movimientos son específicos del deporte, pues son elementos que les ayudan para llegar al máximo rendimiento y mediante un trabajo planificado se alcanzar una técnica especifica. Con este trabajo se pretende llegar al máximo grado de perfeccionamiento y alcanzar objetivos trazados.

Nuevamente se encuentra como en las clases de Educación Física y las sesiones de entrenamiento deportivo se persigue desesperadamente (Sergio, 2007) "el cuerpo-cyborg que es la función máquina organismo y el ideal en esta post- 
modernidad", ya que han quedado evidencias de cómo están impregnadas de la tendencia deportiva e integrada por actividades que fomentan el desarrollo de movimientos relacionados con las capacidades físicas, lo cual hace parte de la gama de la vivencia de la corporeidad. Pero es tan solo una parte de la compleja motricidad humana. En este sentido, (Molúfode, 2009), en términos generales, expresa "Que en las expresiones motrices, se integran una multiplicidad de movimientos que trascienden a acciones políticas, sociales y culturales en las cuales interactúa el sujeto con el otro y el contexto". Con simpatía por las palabras de Molúfode, es importante aportar que las expresiones motrices son la forma en la que el ser humano vivencia y expande su corporeidad en el universo, encarnado en la conciencia, la reflexión, la emancipación, el amor por la vida y la libertad.

Las palabras de la profesora Rubiela y del Grupo de Investigación Molúfode invitan a la reflexión sobre la reducida dinámica en la que vive la Educación Física y el Deporte Escolar, pues se ha evidenciado como el cuerpo y el movimiento humano no sólo se remite a ese ejercicio físico y visible, concentrado en las ansias de aumentar la resistencia, la fuerza, la disciplina, la reciedumbre, la velocidad, entre otras. ¿Por qué no se busca también el amor, la convivencia, el liderazgo?, ¿por qué no se mencionan las emociones y los sentimientos como integrantes de la complejidad humana? ¿Por qué se desprecian las tristezas, las alegrías, los sueños y las frustraciones en las clases y las sesiones de entrenamiento? En fin, ¿Por qué la ceguera ante la compleja motricidad humana?

\section{Bibliografia}

Española, R. A. (s.f.). Diccionario de la Real Academia Española. Concepto de instrumento. Recuperado el 10 de 12 de 2010, de http://buscon.rae.es/drael/ SrvltConsulta?TIPO_BUS $=3 \&$ LEMA=instrumento

Kon-Moción. (2008). Raices costitutivas de la ciencia de la motricidad humana. En Kon-Moción, en-acción 5 Motricidad humana y gestion comunitaria: una propuesta curricular. (págs. 31-52). Popayán: Universidad del Cauca.

Luis Guillermo Jaramillo Echeverri y Otros. (2010). Corporeidad en-acción Experiencia Re-Encarnadora del cuerpo. En Kon-Moción, en-acción 6 Nervaduras la motricidad humana (págs. 31-53). Popayán: Universidad del Cauca.

Merleau-Ponty, M. (1956). Fenomenología de la percepción. Barcelona: Península.

Molúfode, G. d. (2009). Motricidad humana y CONSTRUCCIÓN de Tejido Social en sectores vulnerables "experiencia de investigación. Neiva: Universidad Surcolombiana.

Morris, D. (2000). Docencia y cultura en la era posmoderna. Lisboa: Instituto piaget.

Rey, A., \& Trigo, E. (2000). ¿Quién eres? Apunta N59, 91-98.

Rodriguez, P. (2001). Mentiras Fundamentais da Igreja Católica. Lisboa: Terramar.

Sergio, M. (2007). en-acción 4 Algunas miradas sobre el cuerpo. Popayan: Universidad del Cauca.

Sergio, M., \& Toro, S. (2005). La Motricidad Humana, un corte espitemológico de la Educción Fisica. en-acción 1 consentido, 103.

Teves, N. (2004). Olhares sobre o corpo. Lisboa: Escencies Zubiri, X. (1988). Sobre el hombre. Salamanca: Sigueme. 